Original Article

\title{
Diffused Scanning, Blind Stick \& Rehabilitation
}

Salma Tariq
College of Biomedical Engineering, Ziauddin University, Karachi, Pakistan
Corresponding Author: salmatariqali@ hotmail.com

\section{Abstract}

Objective Pakistan is a developing country and strive to provide cost effective solutions for rehabilitation and aid of visionless individuals with innovative technologies. The purpose of this study is to design and study the blind stick prototype implanted with modern technology. Method Its significant characteristics is embedded photo-electric sensor that uses diffusereflective scanning to sense hindrance and feedback it to the high quality buzzer which can be heard easily furthermore it operates by only rechargeable $9 \mathrm{~V}$ battery. This will enable the blind individuals to sense and detect the obstacle from $5 \mathrm{~mm}$ to $127 \mathrm{~mm}$ distance with simplicity. Result This prototype was used by a blind person in hall with obstacles. Results were tremendous and promising as the blind individual crossed the hall without tripping even a single time. Conclusion It is portable, light weight, user friendly and extremely cost effective that is 4.77\$ (500 Pakistan Rupees) only. It can be used not only in Pakistan but in other least developed countries as well. Blind as well as partially blind person can use it for walking in designated place independently.

\section{Keywords}

Blind Stick Prototype, Photoelectric Sensor, Diffuse Reflective Scanning, Rehabilitation,

Economical.

\section{Introduction}

A study from the Department for International Development (DFID) in 2000 reported that "as many as $50 \%$ of disabilities are preventable". As many of the causes of impairments are preventable, community development organizations in developing countries initiate community-based programs to provide basic prevention and rehabilitation services for persons with disabilities in a "low-cost, but highly accessible manner" 1 . The objective of rehabilitation is to reduce the impact of disabling conditions and to enable persons with disabilities to achieve equal opportunity and full participation in community development activities ${ }^{1}$. Consistent with this objective, disability specialists in the West developed different rehabilitation models to provide basic prevention and rehabilitation services for persons with disabilities.

In developing countries, most persons with disabilities who live in rural areas cannot afford institutional care, and ultimately do not have access to its services as well as government has limited resources to catering services for persons with disabilities. Number of privately owned institutional care and rehabilitation services for persons with disabilities are also available in urban areas only, more over they use modern technologies and therefore services are expensive $^{2}$.

Since centuries, mobility has been the provocative issue for blind people or partially sighted people. Everybody including blind people needs to move. 
Therefore, Blinds since old ages have been using walking stick for their mobility purpose. In addition, some of them are also using guide-dog as well. About $90 \%$ of the blind cannot travel independently; $7 \%$ use a white cane; $3 \%$ could use a Guide dog but only about half of them choose to use a guide dog due to the burden of the Caring for $i^{3}$. Hence there are some limitations with such devices as they are not much efficient. With the advancement of technology, several prototypes have been developed for blind assistance. They are usually known as blind stick. Proposing cost effective and user friendly blind stick prototype which uses diffuse reflective technique also known as "diffuse scanning".

\section{Methodology}

Significant components used are IR emitter, IR receiver, potentiometer and amplifier to form prime photo-electric sensor. Along with it DC battery, power switch and buzzer are connected in a blind stick.

\section{Photoelectric sensor}

A photoelectric sensor is a device used to detect the distance, absence or presence of an object by using a light transmitter and a photoelectric receiver. A photoelectric element can convert light signals into electrical signals using Photoelectric Effect. There are many advantages of using a photo electric sensor such as quick response, simple structure, convenient to use, ability to detect signal without contact and high reliability ${ }^{4}$. There are factors by which detection of target varies. It includes type of sensors, the nature of light and method.

\section{Photoelectric Diffused Mode}

It is also called diffused proximity sensing mode. It is the detection of the sensor's own transmitted light as reflected from the target object's diffusely reflecting surface as shown in fig 1 . These sensors are intended for direct detection of an object rather than indirect detection via beam blocking. The advantage is that they can therefore be used without need for a reflector or detector on the other side of the target object. Both source and detector are on the same side of the object, mounted side by side, usually in same photoelectric housing 5 . In diffuse scanning, transmitter emits light that strikes the object and then reflects the light at random angles. A number of the reflected light comes back to the receiver, and detection of the target is achieved. The transmitter and receiver is placed perpendicular to the target. Casing and positioning is very important. Both transmitter and receiver should be adjusted as well as enclosed in black plastic box to avoid surrounding reflections as shown in fig 1. Both of these factors play important role and aid in proper detection of an obstacle.

\section{Other Features}

The key feature of blind stick is the implantation of photo-electric sensor that sensed the distance from hurdle using diffuse-scanning method. It is portable, cost effective, light weight and operates by only $9 \mathrm{~V}$ battery. This will direct the blind person to the available path. However, the output will be in the form of high quality buzzer which the blind person can hear comfortably.

This model is available with following features:

- On/Off Switch

- Photo-Sensor

- Limited range

- Rechargeable/ Changeable 9V DC battery

- Light Weight

- Device Working 
Figure 1: Diffuse reflective method

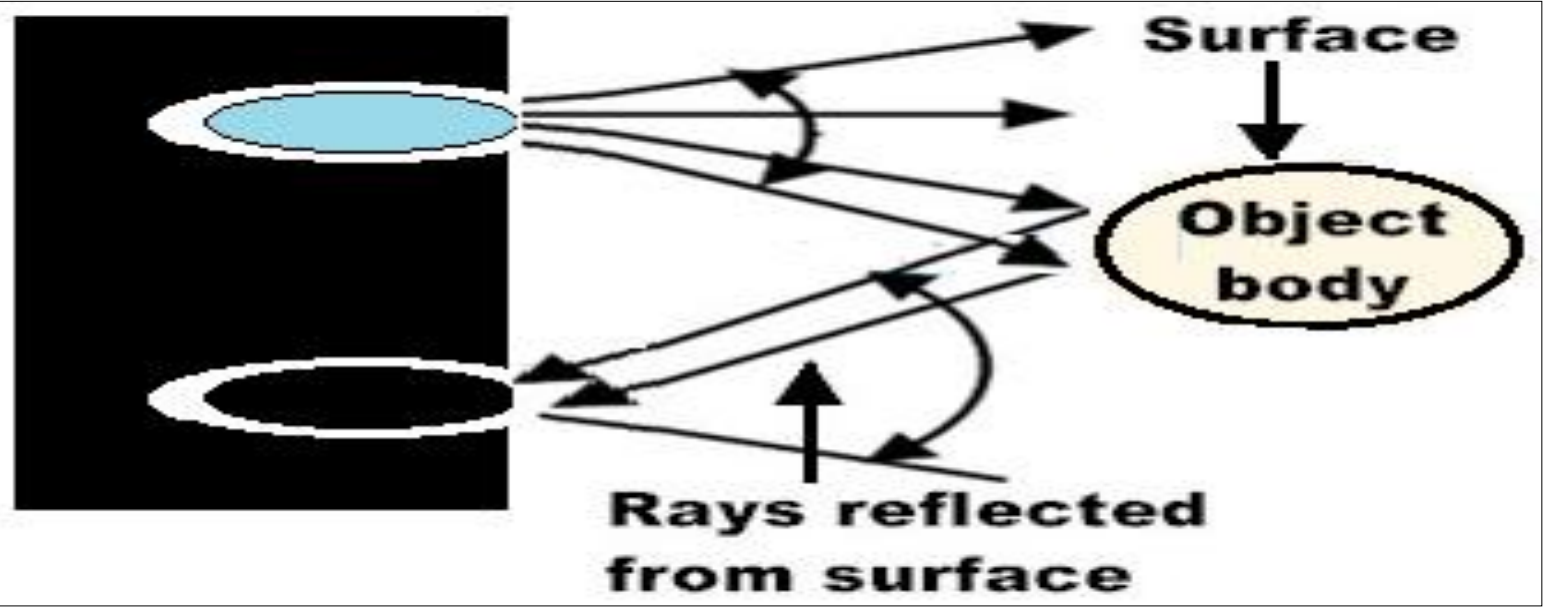

Figure 2: Device Working

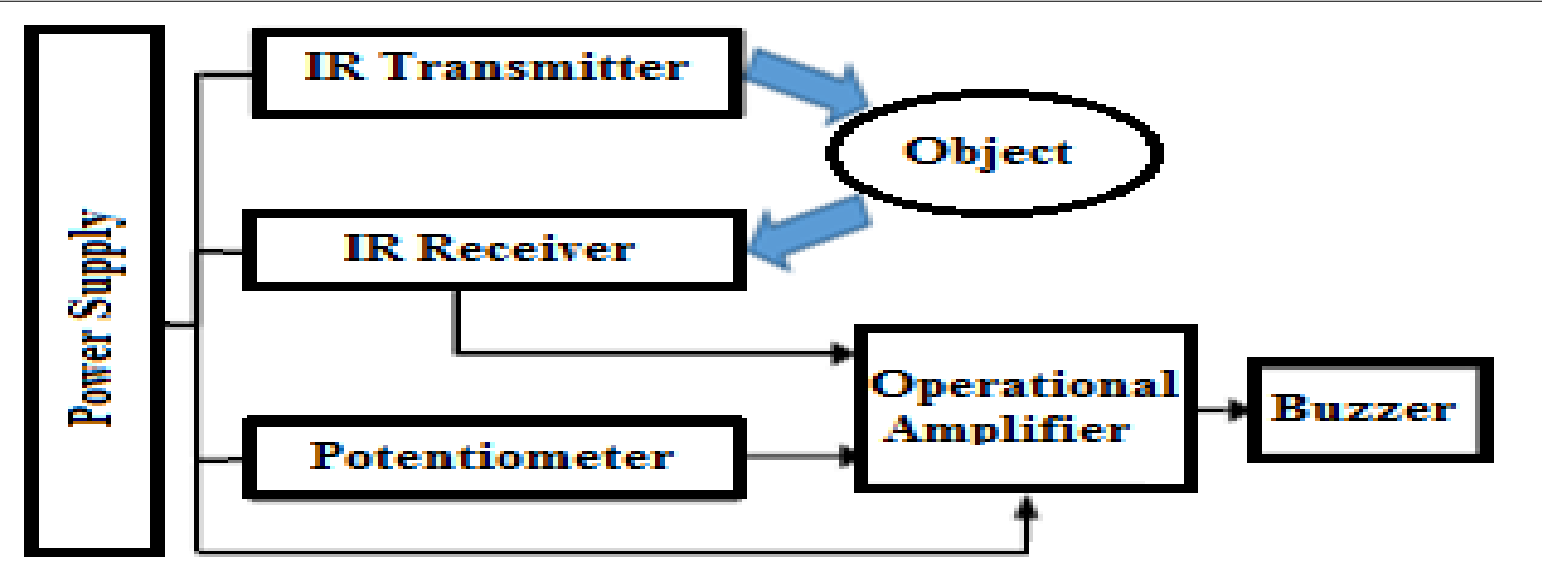

\section{Device Working}

This model employs DC nine volts' battery, a switch is connected between the batteries and photo-electric sensor. Switch can be turn on while using and turn off when not using the stick.

IR LED is used as IR transmitter that emits and photodiode used as IR receiver that detects the infrared light. An amplifier is used here as a voltage comparator and the potentiometer is used for adjustment in the sensing range according to the conditions and requirement.

When the emitted light of IR LED hits an object and reflected back to the photodiode its resistance drops to low value causing a greater voltage flow as compare to voltage threshold of amplifier. As a result, the sensor activates the buzzer and an audio output enables a blind person to recognize the obstacle. 
Physical description of E-smart blind stick

\begin{tabular}{|l|l|}
\hline Stick Color & Black \\
\hline Sensor Range & $127 \mathrm{~mm}$ (5 inches) \\
\hline Length & 40 Inches \\
\hline Weight & $250 \mathrm{~g}$ \\
\hline
\end{tabular}

\section{Experiment}

Through the experiment, we anticipated to identify how well users performed a navigation task with both a white cane and prototype to evaluate the objective utility and effectiveness. The test was conducted in a hall to simulate real-world conditions; many of the obstacles were placed in there randomly. Obstacles were of different shape and materials which include hard and soft materials. A blind person was first allowed to walk in the room for few minutes with his traditional blind stick. Then he was explained about prototype and was asked to take a trial by roaming in hall loaded with obstacle.

\section{Results}

Results were tremendous and promising as the blind individual crossed the hall without tripping even a single time. While walking with traditional white cane, He frequently collided with the objects. Subject described that while using the traditional cane he senses the obstacles and hindrances after reaching near it and sometimes stumbled upon the objects. After using the prototype, he showed positive response. He expressed that he walked very easily because the prototype warned him when he reached the nearby object. It aided him to change his path and check clearance of path by placing a blind stick at each step.

\section{Discussion}

Poor overall health conditions, poverty and malnutrition are major factors resulting in higher rates of disability in many developing countries ${ }^{2,1}$ writes that the "most common causes of childhood disability in these countries are malnutrition of pregnant mother and child, injuries before and during birth, exposure to toxic substances and infectious diseases" 5 .

A large number of children in developing countries are born with a wide range of disabilities. This is due to lack of care and treatment at all levels. It is well-known that institutional rehabilitation services in less developed countries are insufficient as compared to the industrialized west. This leads to an increase in the number of persons with disabilities in developing countries ${ }^{2}$.

The United Nations (UN) has estimated that 5 percent of the world's population has some sort of disabilities while WHO's estimation is 10 percent $^{7,8,9}$.

Whatever the estimation is reported by national and international organizations, there is an immediate need for basic rehabilitation services for millions of persons with disabilities in less developed countries $^{2}$. It is important to note that in these regions the number of persons with disabilities is increasing much faster than the annually added supply of rehabilitation services $^{8}$. Instead, the inequality in the sharing of rehabilitation services in developing countries generates numerous challenges for persons with disabilities particularly those who live in poverty ${ }^{2}$.

Recent research shows that persons with disabilities are systematically excluded from all development activities in many societies. 
This, in turn, limits their rights and challenges their ability to fulfill their socioeconomic obligations ${ }^{10}$.

It is now widely accepted that poverty and disability have reinforced each other and contributed to the increased vulnerability and exclusion of persons with disabilities ${ }^{11}$.

Being aware of this situation, international development organizations and their interest groups have begun to realize that the needs and rights of persons with disabilities cannot be fully addressed unless the underlying causes of poverty are tackled" ${ }^{1,2}$, quoted "the disability specialists have asserted that up to 50 percent of some types of disabilities could be prevented by immunizations, environmental improvements and better overall health care" ${ }^{12}$. The concept of prevention and rehabilitation in the developing world is viewed as significantly important because so many impairments can be avoided $^{2}$. Several prototypes have been developed which incorporated different high end sensors and technologies. They are more efficient and reliable and having more features like GPS, GPRS and Android based system and headphones. But it significantly increases the cost of developing. Such devices are not affordable and economical for majority. Proposed design not only fulfil its basic objective but have most significant feature-inexpensive and reasonable that is 4.77\$ (500 Pakistan Rupees) only. The overall device is ideal for blind individuals. When manufactured on large scale the price can reduce to approximate 200 Pakistani Rupees. It can be used not only in Pakistan but in other least developed countries as well.

\section{Conclusion}

The main purpose of this study is to design and study the blind stick prototype. From the tests carried out on its functions reveal that the developed prototype has achieved its objective efficiently. Results are tremendous and promising as the blind individual crossed the hall without tripping even a single time. It is portable, light weight, user friendly and extremely cost effective. It will help in rehabilitation of blind individuals not only in Pakistan but all around the world especially in poor and third world countries where resources and rehabilitation is quite limited to specific individuals.

\section{Conflict of Interest}

No competing interests were found in the preparation of this paper.

\section{Acknowledgement}

I would like to acknowledge all my teachers who encourage me and support me in writing of this paper, furthermore to all the researchers and healthcare professionals (along with all the private institutes, funding agencies and NGO) who strive and give their efforts in rehabilitation of disabled and handicapped throughout the world.

\section{References}

1. Lightfoot, E. (2004). Community-based rehabilitation a rapidly growing method for supporting people with disabilities. International Social Work, 47(4), 455$468+559+561+563+565+567$.

DOI: $10.1177 / 0020872804046253$

2. Md.Hossain (2008). Community Approaches to Handicap in Development (CAHD): Strategy to Implement Community-based Rehabilitation in Less Developed Countries, p.10-12+14+22.

3. Mau.S, Melchior.N.A, Makatchev.M and Steinfeld.A May, (2008) Blind Aid: An Electronic Travel Aid for the blind, tech. report CMU-RI-TR-07-39, pp.6. 
Retrieved from Robotics Institute, Carnegie Mellon.

4. Wang.P and Liu.Q (2011). Photoelectric sensors. In Biomedical Sensors and Measurement (chapter 3).

5. Juds.S.M (1988). Diffuse Proximity Sensing. In Photoelectric Sensors and Controls: Selection and Application (Chapter 3, First Edition).

6. Hammerman, S. (1986) 'Disabled People in the Third World: The Silent Emergency', in D. Warms (ed.) Into the 21st Century: Selected Papers from the UCP International Conference on The Changing Rehabilitation World, pp. 1038. New York: United Cerebral Palsy of New York City.

7. Alam, Jahurul. Khandakar\& Bari, Nazmul. (2005). Community-based rehabilitation: Practices and alleviation of poverty of people with disabilities in Bangladesh. Bangladesh: The National Forum of Organizations Working with the Disabled (NFOWD).
8. Canadian Network on Disability and Development (CNDD). (2006). Disability and development. Toronto: Canadian Network on Disability and Development (CNDD).

9. World Health Organization. (1992). The work of WHO 1990-1991: Biennial report of the director-general. Geneva: WHO.

10. Nagata, K. K. (2007). Perspectives on disability, poverty and development in the Asian region. Asia Pacific Disability Rehabilitation Journal, 18(1), 1-13.

11. Khan, Noman \& Bari, Nazmul. (2002). Disability in development: From charity to equity people with disabilities in Bangladesh. Journal for International Development, 13(1), 100-105.

12. Lambo, T. and N. Sartorius (1990) 'Mental Health Programs: An International Perspective', in T. Lambo and S. Day (eds) Issues in Contemporary International Health. New York: Plenum Medical Book Company. 